increased expenditure and effort on improved ventilation and containment of the source of radon.

As this survey shows, there is wide divergence of views on the legislation which is necessary to ensure the safe use of ionizing radiations. It will be surprising if a future edition of Protection Against Ionizing Radiations does not also reveal a greater diversity of opinions on the acceptable limits of dose which reflect more closely the needs of individual countries.

\title{
J. VENNART
}

\section{MULTIPLE HIT FREQUENCIES IN FULLY CORRELATED FIRE}

\author{
By E. R. TERRY
}

Technical Operations Incorporated, Burlington, Massachusetts

$I^{N}$ evaluating the effectiveness of high-firing-rate guns as air defence and air-to-ground weapons we are often interested in hit frequencies other than the 'no hits', or miss, frequency. Assuming the aimpoint does not move during the firing interval, we can readily determine other hit frequencies by an extension of the 'salvo' or 'shotgun' formula. More precisely we will develop a model for determining the probability of exactly $m$ hits with $n$ rounds when the distribution of shots is circular normal and centred at a point $h$ units from the target origin.

Assume that the pattern of weapons is released at a circular target of radius $R$ with a bivariate circular normal aiming error characterized by a standard deviation $\sigma_{A}$. Each weapon in the pattern is distributed about the mean point of impact with a bivariate circular normal ballistic dispersion and is characterized by a standard deviation $\sigma_{B}$. The ballistic dispersion for each of the weapons comprising the pattern and the aiming error are mutually independent.

The probability that the $i$ th round strikes the target of area $A$ is:

$$
p_{i}=\frac{1}{2 \pi \sigma_{B}^{2}} \iint_{A} \exp \left[-\frac{1}{2 \sigma_{B}^{2}}\left((x-h)^{2}+y^{2}\right)\right] \mathrm{d} x \mathrm{~d} y
$$

Transforming to polar co-ordinates we obtain:

$$
\begin{array}{r}
p_{i}=\frac{1}{2 \pi \sigma_{B}^{2}} \int_{\theta=0}^{2 \pi} \int_{r=0}^{R} \exp \left[-\frac{1}{2 \sigma_{B}^{2}}\left(r^{2}+h^{2}-\right.\right. \\
2 r h \cos \theta)] r \mathrm{~d} r \mathrm{~d} \theta
\end{array}
$$

Rewriting equation (2) as follows:

$$
\begin{aligned}
p_{i}=\left(\frac{1}{\sigma_{B}^{2}} \exp \left[-\frac{h^{2}}{2 \sigma_{B}^{2}}\right] \int_{r=0}^{R} r \exp \left[-\frac{r^{2}}{2 \sigma_{B}^{2}}\right]\right)\left(\frac{1}{2 \pi} \int_{\theta=0}^{2 \pi}\right. \\
\left.\quad \exp \left[\frac{r \cos \theta}{\sigma_{B}^{2}}\right] \mathrm{d} \theta\right) \mathrm{d} r
\end{aligned}
$$

we see:

$$
\frac{1}{2 \pi} \int_{\theta=0}^{2 \pi} \exp \left[\frac{r h \cos \theta}{\sigma_{B}^{2}}\right] \mathrm{d} \theta=J_{0}\left[\frac{i r h}{\sigma_{B}^{2}}\right]=I_{0}\left[\frac{r h}{\sigma_{B}^{2}}\right]
$$

which is the zero-ordered Bessel function of pure imaginary argument. Accordingly equation (3) becomes:

$$
\begin{aligned}
p_{i}=\left(\frac{1}{\sigma_{B}^{2}} \exp \left[-\frac{h^{2}}{2 \sigma_{B}^{2}}\right] \int_{r=0}^{R} r\right. & \\
& \left.\quad \exp \left[-\frac{r^{2}}{2 \sigma_{B}^{2}}\right]\right)\left(I_{0}\left[\frac{r h}{\sigma_{B}^{2}}\right]\right) \mathrm{d} r
\end{aligned}
$$

Since the ballistic dispersions of all the rounds comprising the burst are independent of one another, the conditional probability that exactly $m$ weapons will hit the target if a pattern of $n$ weapons is released is $\left(\begin{array}{c}n \\ m\end{array}\right) p_{i}^{m}\left(1-p_{i}\right)^{n-m}$ if we assume the resulting hit frequencies are binomially distributed. To obtain the unconditional probability that the centre of the distribution of shots is a distance $h$ units from the target centre and that exactly $m$ weapons will hit the target, we must multiply the expression above by the probability of the centre of this distribution of shots being so located.

The probability density function for determining whether the centre of the distribution of shots is within an annulus of width $\mathrm{d} h$ at a distance $h$ units in any direction from the centre of the target is :

$$
\mathrm{d} p_{h}=\frac{1}{\sigma_{A}^{2}} \exp \left[-\frac{h^{2}}{2 \sigma_{A}^{2}}\right] h \mathrm{~d} h
$$

Multiplying equation (6) by the conditional probability that if the centre of the distribution of shots is so located exactly $m$ rounds will hit the target, we get the corresponding probability density function:

$$
\begin{aligned}
\mathrm{d} p_{[n, m]} & =\mathrm{d} p_{h}\left(\begin{array}{c}
n \\
m
\end{array}\right) p_{i}^{m}\left(1-p_{i}\right)^{n-m} \\
& =\frac{1}{\sigma_{A}^{2}} \exp \left[-\frac{h_{i}^{2}}{2 \sigma_{A}^{2}}\right]\left(\begin{array}{c}
n \\
m
\end{array}\right) p_{i}^{m_{i}}\left(1-p_{i}\right)^{n-m} h_{i} \mathrm{~d} h_{i}
\end{aligned}
$$

where the $p_{i}$ are a function of the $h_{i}$. Integrating equation (7), we obtain for the unconditional probability that exactly $m$ weapons will hit the target when a burst of $n$ rounds is fired:

$P_{[n, m]}=\frac{1}{\sigma_{A}^{2}} \int_{0}^{\infty} \exp \left[-\frac{h_{i}^{2}}{2 \sigma_{A}^{2}}\right]\left(\begin{array}{c}n \\ m\end{array}\right) p_{i}^{m}\left(1-p_{i}\right)^{n-m} h_{i} \mathrm{~d} h_{i}$

Equation (8) can be evaluated numerically by employing the Laguerre-Gauss quadrature formula.

The model outlined here has been programmed in Fortran for the IBM 7090/7094. Tables for facilitating a quick solution of equation (8) for any $m$ and $n$ are at present being compiled and will be submitted for publication in the near future.

\section{OPTICAL PROPERTIES OF URANIUM OXIDES}

\author{
By Dr. J. M. JONES and DR. D. G. MURCHISON \\ Department of Geology, University of Newcastle upon Tyne
}

$\mathrm{W}$ E were recently asked to investigate the optical properties of uranium oxides in the composition range $\mathrm{UO}_{2 \cdot 00}-\mathrm{UO}_{2 \cdot 25}$ as part of a research programme being carried out by the Reactor Group at the U.K.
Atomic Energy Authority Establishment, Dounreay, Scotland. It was hoped to establish: (1) that a satisfactory correlation existed between the optical properties of the oxides and their oxygen/metal ratios; (2) if this were the 